\title{
Design of Dynamic Resource Allocation Scheme for Real-Time and Non-Real-Time Traffics in The Advanced Mobile Communications Network
}

Yi-Ting Mai ( $\square$ wkb@wkb.idv.tw)

National Taiwan Sport University https://orcid.org/0000-0003-3580-4001

Chih-Chung Hu

National Chi Nan University

\section{Research}

Keywords: 4G/5G, Scheduling, cross-layer QoS, EURA, resource allocation

Posted Date: July 28th, 2021

DOl: https://doi.org/10.21203/rs.3.rs-699648/v1

License: (c) (1) This work is licensed under a Creative Commons Attribution 4.0 International License.

Read Full License 
Design of Dynamic Resource Allocation Scheme for Real-time and Non-real-time Traffics in the Advanced Mobile Communications Network

Yi-Ting Mai ${ }^{\mathrm{a}, *}$, Chih-Chung $\mathrm{Hu}^{\mathrm{b}}$

${ }^{a}$ Department of Sport Management, National Taiwan University of Sport,

Taichung City, Taiwan, R.O.C.

${ }^{\mathrm{b}}$ Department of Computer Science and Information Engineering, National Chi Nan University,

Puli, Nantou County, Taiwan, R.O.C.

*Corresponding Author: Yi-Ting Mai; E-mail: wkb@ntus.edu.tw,wkb@wkb.idv.tw, wkb@ieee.org

\begin{abstract}
The 5G wireless technology is recently standardized for meeting intense demand. The Long Term Evolution (LTE) technology provides an easy, time-saving, and low-cost method for deploying a 4G/5G network infrastructure. To support multimedia service and higher bandwidth data delivery, an LTE MAC layer has QoS support with several QoS class indicator (QCI) levels. Based on LTE current QCI priority and QoS requirements in UEs, the original Max-Rate scheduler or Proportionally Fair (PF) algorithm could not achieve their goal owing to the UE's dynamic physical capacity with a different channel quality indicator (CQI) at run time. For better QoS service than LTE networks, per UE's CQI state for each resource block (RB) must be considered simultaneously in LTE MAC layer resource allocation with crosslayer support. As DL real estimated capacity is dynamic owing to a UE's periodic CQI reporting, the CQI state in LTE scheduling must be considered. This study proposes a smart and flexible scheme for Enhanced Utilization Resource Allocation (EURA) including three novel mechanisms that can dynamically fit UEs' CQI states. The simulation results in this study demonstrate that the proposed EURA scheme outperforms the contrast schemes, can save more rare radio capacity, and improve the utilization of radio resource assignment.
\end{abstract}

Keywords: 4G/5G; Scheduling; cross-layer QoS; EURA; resource allocation

\title{
1. Introduction
}

Wireless networks are rapidly evolving from $2 G(G S M)$ telephony networks to $3 G$ (UMTS) and beyond. Following the blooming markets of cellular phone networks and popular Internet services, mobile highbandwidth data communication is becoming a new and promising business niche. For many network connection requirement users, it is the basic target for advanced wireless technology venders to provide wireless network services anytime and anywhere. Therefore, wireless technology for the fourth generation $(4 G)$ of wireless broadband communications has been standardized in recent years. Long Term Evolution (LTE) and LTE-Advanced (LTE-A) [1]-[6] is commercially deployed in many countries. LTE offers traditional voice telephone service and provides a cost-effective broadband communication service. The Third Generation Partnership Project (3GPP) formally recognizes the LTE platform as the technology standard for wireless communications. Since LTE standard is defined by telecom vendors and is backward compatible with the GSM or UMTS cellular systems, its deployment is much easier than with traditional IEEE wireless network technology. Moreover, the latest fifth-generation (5G) combined 
with D2D communication technology is used to improve transmission quality for users, and it achieves better performance even in high-speed movement. Therefore, the $5 \mathrm{G}$ new radio $(N R)$ international standard 3GPP has already regarded D2D as an extremely important application scenario in the communication technologies of R15 [7][8] and R16 [9][10]. At present, 4G LTE networks and 5G NR cooperate with each other and provide mobile network services. MAC layer data scheduling in 4G LTE and 5G NR has many technologies in common. This study first discusses how to design a mechanism to allow data transmission scheduling to concurrently support QoS within a MAC layer in an LTE architecture when the real radio capacity of multiple mobile users changes dynamically.

Based on GPRS (2.5G) and UMTS network technologies, both the core network (Evolved Packet Core, EPC) and radio access (Evolved Universal Terrestrial Radio Access Network, E-UTRAN) in LTE are completely packet-switched models. Moreover, LTE is designed to work with different bandwidths and provide a peak data rate at $100 \mathrm{Mbps}$ in a downlink and $50 \mathrm{Mbps}$ in an uplink. To support multimedia services and high-bandwidth data delivery, an LTE MAC layer supports QoS with different QoS class indicator (QCI) levels. Therefore, some researchers have tried to adopt the maximum throughput (MT) [11][12] as Max-Rate scheduler or Proportionally Fair (PF) [13] algorithm as LTE MAC scheduling scheme in Evolved Node $B(e N B)$ to maximize throughput or allocate a fairness bandwidth for many mobile users. However, based on LTE current QCI priority and QoS requirement in UEs, the original MT scheduler cannot achieve maximization throughput due to a run-time UE's dynamic capacity with a variation channel quality indicator (CQI). In addition, although the traditional PF algorithm can achieve the fairness of the rate between different UEs, and the reference of rate corresponds to the number of subRBs, in a wireless network environment, the same number of RBs may occur owing to changes in CQI over time. However, an actual UE's assigned rate cannot reflect an actual situation.

For providing better QoS service over LTE networks, per UE's CQI state for each resource block (RB) should be considered simultaneously in LTE MAC layer resource allocation (Figure 1). It is necessary to consider the CQI state in LTE eNB scheduling. The Enhanced Utilization Resource Allocation (EURA) scheme has been proposed in this study. Furthermore, three novel mechanisms in the proposed EURA scheme can dynamically fit UEs' CQI state. The proposed EURA scheme with three mechanisms can improve the utilization of an LTE radio resource.

This study is organized as follows. LTE MAC technology and some scheduling in an LTE network are briefly introduced in Section 2. Method of the proposed EURA scheme for LTE is presented in Section 3. Results, simulation study, performance comparison and discussion are listed in Section 4. Finally, we will conclude this study in Section 5.

\section{Related work}

\subsection{LTE MAC Layer}

The basic time unit for packet scheduling and transmission in LTE network is called a transmission time interval (TTI) with a length of $1 \mathrm{~ms}$. Thus, TTI is the time unit for LTE resource allocation. In each TTI, a scheduling decision is made, in which each scheduled UE is assigned a certain number of radio resources 
in the time and frequency domain. In the time domain, a TTI is split into two slots (one slot is $0.5 \mathrm{~ms}$ ). Each slot comprises seven OFDM symbols in the case of the normal cyclic prefix length. In the frequency domain, resources are grouped in units of 12 subcarriers, such that of one unit of 12 subcarriers for a duration of one slot is called an $\mathrm{RB}$, which is the smallest element of resource allocation. The smallest unit of a resource is a resource element (RE) that consists of one subcarrier for a duration of one OFDM symbol. Therefore, an RB is comprised of $84(7 \times 12)$ REs in the case of the normal cyclic prefix length in Figure 2.

The channel capacity was assumed to be static for traditional MAC scheduling, and it was revised for LTE network environments. In LTE, an eNB typically selects the modulation and coding scheme (MCS) depending on a prediction of the DL channel condition, which is according to the UE's CQI report transmitted (Figure 1). The 3GPP LTE has given a table of references for the efficiency of each CQI index (CQI ranges from 1 to 15 by the modulation type of 64QAM, 16QAM, and QPSK) as Table 1.

\subsection{Related research}

In the field of $4 \mathrm{G} / 5 \mathrm{G}$ wireless network research, the research on the scheduling of various MAC layer networks has always been the focus of many researchers, such as the research on handover scheduling in homogeneous or heterogeneous mobile networks [14][15] and research on multimode quality of service $(Q o S)$ guarantee [16]-[22]. In the LTE mobile network scheduling research field, some research focus on the discussion of uplink scheduling [23][24], but most of the research focus on downlink scheduling. In addition, to allow mobile users to allocate resources within the limited wireless network bandwidth, it is necessary to incorporate QoS considerations in scheduling.

Ali et al. [25] and Jang et al. [26] had proposed different policies for different QoS data traffic types to improve QoS performance, better delay guarantee, and high throughput. Biernacki et al. [27] proposed some fairness algorithms to find a balance between different QoS type traffic to avoid starvation at lower QoS level traffic flows. Considering the scheduling algorithm and QoS support at the same time, Aminu et al. [28] conducted a survey on many scheduling algorithm mechanisms for the LTE MAC layer scheduling mechanism and characteristics of QoS considerations. Some comparative analyses have been performed on their research results to explore the various parameters of the various downlink scheduling algorithms for resource allocation. Deniz et al. [29] mainly used the mobile user (UE) role at the edge to further explore different resource allocation scheduling algorithms. In the consideration of throughput and fairness, the advantages and disadvantages of each are discussed. The mechanism proposed by the author can simultaneously consider two performance parameters and have fairly good performance results. Nasralla et al. [30] discussed and analyzed many current QoS-aware downlink scheduling algorithm mechanisms in LTE networks and divided these mechanisms into four main classes, namely, delay aware, queue aware, target bit rate aware, and hybrid aware. They also propose to use the hybrid aware category as a conceptual mechanism to propose a resource allocation scheduling algorithm that considers QoS while also taking into account fairness. In doing so, there can be a certain degree of scheduling fairness in the face of real-time and non-real-time traffic.

However, the relation between QoS level and UE's CQI state is quite important for QoS supporting. How 
to improve the LTE overall system performance under fairness, channel state, and bandwidth constraints is a complicated job for the eNB allocates the appropriate resources for many UEs in each TTI. For providing better QoS service over LTE networks, per UE's CQI state for each RB should be considered simultaneously in LTE MAC layer resource allocation (Figure 1). However, the effective use of scarce radio resources has not been further discussed in detail in previous studies. This study will further explore this part.

\section{Methods: Enhanced Utilization Resource Allocation (EURA) scheme}

Estimation of the channel capacity depends on the CQI reports from a UE, meaning that different UEs would have different views of the channel capacity (e.g., RB1 to RB6, as bottom part of Part 0 of Figure 3). Since current per RB capacity via each UE is dynamic, it is important to schedule these DL RBs for meeting a UE's requirement appropriately. For LTE resource allocation in the MT scheduler with the priority queue scheme, the higher priority level of QCI-1 data (UE1 in Figure 3) must be scheduled first. For example, the idea capacity of the dotted line of RB is 120 bits; however, the real RB capacity depends on each UE's CQI report, which is the solid line of RB as illustrated blocks in Figure 3. Therefore, the 100 bits capacity of RB1 is allocated to the UE1's data requirement as Part 1 of Figure 3. Then, to fit the respective UE2 200 bits and UE3 70 bits data (in Figure 3) will require the lower priority level, QCI-2. All the remaining RBs (RB2, RB3, RB4, RB5, and RB6) also must be assigned as Parts 2-6 of Figure 3. Finally, the case of three UEs' resource allocation scenario is shown in Figure 3. In the example, there are six assigned RBs at the adopting scheme of the MT scheduler with a priority queue. Some problems are listed as $P 1, P 2$, and $P 3$ in Part 6 of Figure 3. The highest capacity size RB (RB1 for UE2) cannot be allocated as $P 1$ problem, no choosing policy when assigning RB3 or RB6 for UE2 as $P 2$ problem, it might save one free RB5 when assigning RB6 for UE2, and RB3 for UE3's total 70 bits data requirement; moreover, some remaining useless capacities can be found in RB4 with UE2 and RB6 with UE3 as P3 problem. To allocate RB resources with some policies will improve the utilization of RBs allocation in LTE networks.

\subsection{Basic idea}

Since each UE DL real capacity is dynamic owing to UE's periodical CQI reporting, it is necessary to consider state in LTE scheduling. Our proposed EURA scheme can dynamically fit UEs' CQI state to improve the performance of LTE wireless capacity. Initially, if the total UE requirement data is not in heavy load state, the QCI priority queue rule will not need to be adopted as dynamic QCI level adopting checking policy in our proposed EURA scheme. For RBs allocation, the better UE CQI state in RBs should be assigned in advance to prevent the $P 1$ problem in Figure 3. The first idea of the proposed mechanism is Larger $R B$ capacity first. Second, if there are more than one suitable RBs for selection, lower not available RB capacity (i.e., the lower total remaining space of other UEs in this RB) would be assigned first to reduce future side effect as $P 2$ problem in Figure 3. The second idea of the proposed mechanism is lower remaining $R B$ space first. Finally, if there are two or more $\mathrm{RB}$ candidates for assignment, fitting the UE's data requirement should be assigned first, which might decrease occurrence rate as $P 3$ problem in Figure 3. The third idea of the proposed mechanism is lower useless RB capacity first. For regular LTE 
resource allocation, our proposed EURA scheme includes these one checking policy and three novel mechanisms in Figure 4. The details of mechanisms are in the next section.

\subsection{EURA}

An optimal RBs allocation solution EURA is proposed in this study without using traditional QCI level option in the condition of which the total UEs data requirement is lower than radio deliver capacities. When the total data amount to be transmitted is smaller than the total of the RB capacities, QCI level is not necessary for LTE MAC scheduling as illustrated in Part 0 of Figure 5. Using EURA, the largest amount of data in a UE buffer is allocated to one of the RBs. If the largest data in this UE is greater or equal to one of the RB that has the largest space, the data is sent to this RB; otherwise, it is sent to one of the smallest RBs that has enough space for data from a UE buffer. In Part 0-1 of Figure 5, the largest amount data is 200 bits in UE2 and 120 bits of them are transmitted to RB1 that has 120 bits space, which is the largest one. The remaining 80 bits data are left in UE2. Further, the maximum amount of data is 100 bits in UE1. RB4 has 110 bits space for UE1, but it is not the smallest one. Therefore, data in UE1 is then transmitted to RB5 that has 100 bits capacity as shown in Part 1-2 of Figure 5. To prevent other UEs from having the opportunity to use higher RB space in the future so 80 bits in UE2 is sent to RB6 rather than RB3. Finally, the 70 bits in UE3 is transmitted to RB3.

The EURA algorithm is summarized as follows.

- EURA can be applied without the priority of QCI level when the total data amount to be transmitted is smaller than total of the RB capacities based on the Dynamic QCI level adopting policy.

- The maximum amount of data in one of the UE buffers is allocated to one of the RBs in a group.

- If the largest data in this UE is more than or equal to one of the RB capacities that has the largest space, the data is sent to this RB in this TTI, and it is the Larger RB capacity first mechanism.

- If the largest data in this UE is less than or equal to at least one RB capacity, data is sent to the smallest unallocated $\mathrm{RB}$, and it is the Lower remaining $R B$ space first mechanism.

- If there are two or more available candidate RBs, to choose the RB that can fit the UE's data requirement with lower other UE's RB useless capacity should be assigned first, it is the Lower useless RB capacity first mechanism.

Compared with the MT scheduler with the priority queue scheme (Figure 3), our proposed EURA scheme with three mechanisms will have two more free RBs space for the abovementioned case (as shown in Figure 5). Therefore, our proposed scheme can improve RBs utilization. It would also save more RB space for further resource allocation in $4 \mathrm{G} / 5 \mathrm{G}$ networks.

\subsubsection{Dynamic QCI level adopting}

Since the channel capacity varies owing to the adaptively selected MCS for each UE. The estimation of the channel capacity for $R B_{i}$ from $U E_{j}$ 's CQI report as $C Q I_{i, j}$. In CQI report, the UE reports a CQI value 
for each RB. Notations used in the estimation of the all RB capacity for $U E_{j}$ are defined as follows and also illustrated in Figure 6.

$N_{O F D M}^{T I I}$ : The number of OFDM symbols (REs) in a TTI that are 14 in the case of the normal cyclic prefix length.

$N_{O F D M}^{C r l}$ : The number of OFDM symbols used by the control channels in a TTI.

$N_{O F D M}^{R e s v}$ : The number of OFDM symbols reserved for reference signals in a TTI of 12 subcarriers.

Note that the two RBs in a TTI of 12 subcarriers are called the $R B$ group $(R B G)$ in the study. Therefore, the number of REs for the user data in an RBG, denoted by $N_{R E}^{T T I}$, is calculated as follows.

$$
N_{R E}^{T T I}=\left(N_{O F D M}^{T T I}-N_{O F D M}^{C r r l}\right) \times 12(\text { subcarriess })-N_{O F D M}^{R e s v}
$$

For the CQI report, each RB channel capacity estimated for $U E_{j}$ in an $R B G_{i}$, denoted by $C_{i, j}^{R B G}$, is calculated as follows.

$$
C_{i, j}^{R B G}=N_{R E}^{T T I} \times E f f\left(C Q I_{i, j}\right)
$$

The function of $E f f\left(C Q I_{i, j}\right)$ in $E q$. (2) returns the efficiency value for the given CQI value $C Q I_{i, j}$ according to Table 1 in the system. Based on this, $N_{R B G}$ is the total number of RBG in the system.

For the case of the full-Sub-band report, the channel capacity estimated for $U E_{j}$ in a TTI, denoted by $C_{j}^{\text {Report }}$, is calculated as follows.

$$
C_{j}^{\text {Report }}=\sum\left(N_{R E}^{T T I} \times E f f\left(C Q I_{i, j}\right) \times N_{R B G}\right)
$$

As the estimation of the traffic load for a UE, the estimation of the current channel capacity for $U E_{j}$, denoted by $C_{j}$, is calculated by exponentially averaging the samples of each calculation. The channel capacity for all UEs is calculated by combing the channel capacity estimated by each UE with the ratio of the UE's traffic load in the group. The channel capacity (bits/TTI) for all UEs, denoted by $C_{\text {Channel }}$, is calculated as follows: 
$C_{\text {Channel }}=\sum_{\forall U E_{j}}\left(C_{j} \frac{\lambda_{j}}{\lambda}\right)$, where $\lambda$ is the total DL load, and $\lambda_{j}$ is the current load of $U E_{j}$. Because we can use the Eq. (4) to calculate the average data rate that all RBs of the current TTI can provide, and through a weighted value $\beta$ in the formula, we can make dynamic adjustments.

$$
S_{c h} h_{T h r}=\beta \times C_{\text {Channel }}
$$

Thus, we can refer to the first step "Load state/Capacity estimation" in our proposed EURA scheme, as shown at the top of Figure 4. We compare the current usable bandwidth space calculated using the $E q$. (4) with the amount of all UE buffer data in each TTI period in the eNB. If the bandwidth is sufficient (Figure 4, "Dynamic QCI level checking" and "Yes") as the result of Eq. (5). In fact, at this time, not necessary to consider the QCI weights between different UEs, i.e., all UEs are regarded as the same priority group so that the overall radio resources can be used most effectively.

$$
S c h_{T h r} \geq B u f f e r_{U E_{-} \text {Data }}
$$

\subsubsection{Larger RB capacity first}

If there are more than one UEs groups after the dynamic QCI level adopting procedure, the allocated sequence based on group priority. In a UEs group, we will schedule according to the amount of UE buffer data, and the largest amount of buffer data will give priority to the allocation of RB radio resources. When the highest priority UE has many RB candidates can be selected for resource allocation at the same time, the largest RB will be effectively allocated to achieve the goal of high-quality RB space allocation in advance in Part 1 of Figure 5.

\subsubsection{Lower remaining $\mathrm{RB}$ space first}

To find an appropriate RB assignment rule is very important for improving $\mathrm{RB}$ resource allocation in our proposed EURA scheme. When it is necessary to allocate the highest UE buffer data volume, if there are multiple RBs whose capacity is greater than or equal to the space requirement of the UE buffer, we should give priority to one sufficient RB space to avoid leftovers. The main reason is that one RB can only be allocated to one UE in the same TTI, and this mechanism is called Lower remaining RB space first. Through this mechanism, the precious RB radio space can be used very effectively. In Part 1 and Part 2 of Figure 5, the RB5 will be allocated for UE1 to avoid 10 bits useless space for RB4 allocation; furthermore, the remaining data of UE2 will be assigned to RB3 or RB6 as there would be 30 bits useless space for RB2 assignment.

\subsubsection{Lower useless RB capacity first}

A better allocation decision for LTE MAC scheduling could increase DL utilization when there are two or more free RB candidates for resource allocation. Our proposed Lower useless RB capacity first mechanism would reduce the issue of wasted RB resource in the next schedule. When UE buffer data needs to be allocated, if there are more than two RBs with the same RB capacity to choose from, we will give priority to the RB that is poor in quality compared to other UEs because it has less capacity. This 
will avoid the need to use more RBs when scheduling in the future. Moreover, according to the 80 bits in UE2 to be transmitted, there are four RBs candidates as RB2, RB3, RB4, and RB6 (Part 2 of Figure 5) can be chosen. According to the principle of "lower remaining RB space first" in Section 3.2.3, we will only consider RB3 and RB6. When it is all 80 bits, each RB cannot be used by other UEs after it is selected for one UE. Therefore, the least unusable space, in theory, is allocated by RB6 (RB3 90+70, RB6 80+50) as shown in Part 3 of Figure 5. However, if we do not adopt this mechanism, suppose RB3 is allocated to UE2 instead, and when the subsequent UE3 is to be allocated, two of RB2, RB4, and RB6 must be selected to transmit all the data of UE3.

\section{Results and Discussion}

\subsection{Experiment setup and performance criteria}

In Figure 7, the simulated network environment consists of one LTE-A eNB and maximum 20 UEs. Each UE traffic flow contains audio and video and has different packet sizes for different types of traffic flows. Different UEs present the level of a traffic load. In this simulation environment, the theoretical load value is $\rho=0.25$, number of UEs is 20 , and each RB can provide the optimal ideal value capacity of CQI-15 in Table 1 for calculation. However, the load in the actual simulation environment will be higher than usual. The main reason for this is that each $\mathrm{RB}$ will be calculated based on the changes in the actual reported CQI due to the different locations of the UEs. Therefore, the RB capacity that can be actually used must be much smaller than the ideal value. In the simulation results, we can find that traffic load $\rho>0.9$ in the heavy load situation when $U E=20$. In the dynamic QCI level adopting mechanism in Section 3.2.1, we set the parameter $\beta$ in the threshold of $S c h_{T h r}$, and what would be a better value for parameter $\beta$ ? Estimation it in a simulated experimental environment, we assume that $\beta$ is $1-2$. When $\beta$ is 1 , it means that there is a $50 \%$ theoretical probability that $E q$. (5) is found, and when $\beta$ is 1.2 , it is $60 \%$, when $\beta$ is 1.4 , it is $70 \%$, and when $\beta$ is 1.6 , it is $80 \%$. When $\beta$ is 1.8 , it is $90 \%$, and when $\beta$ is 2 , it is $100 \%$. By estimating the experimental hit rate results as shown in Figure 8, we found that setting $\beta$ to 1.8 yields the highest success rate; therefore, in our experiment, we will use $\beta=1.8$. Other simulation parameters are shown as Table 2. A simulation study is conducted to evaluate the performance of our proposed EURA scheme The MaxRate scheduler [12] can have MT feature and the EXP/PF [27] can have fair allocation schedule with lower delay so that the two contrast schemes are also simulated. For the EURA algorithm we proposed, we mainly hope to improve the utilization of the entire RB and prevent the delay of the UE from increasing considerably. Therefore, in the simulation, the main goal is to understand the use of RB capacity in the entire resource allocation. In addition, delay and throughput for commonly used traffic flow are also topics that we need to discuss. The five parameters in the simulation experiment are described as follows.

- Residual RB (\%) that is the average percentage of unallocated number of DL RB in a TTI.

- Residual Capacity (bits) that is the average of total unallocated DL RBs' bits in a TTI.

- Rate of waste capacity (\%) that the average percentage of allocated DL RB's useless bits in a TTI is also defined as the difference between RB real capacity and an assigned UE's data. Furthermore, it is also the P3 problem (a case of Part 6 of Figure 3 for the RB4 and RB6). 
- Delay $(m s)$ that is the average system time of a UE's DL traffic data at eNB buffer.

- Total Throughput (kbps) that is the receive traffic flow bit rate for all UEs in the simulation.

\subsection{Experiment Results}

There are three parts for performance evaluation. Initially, the simulation must identify performance and resource allocation utilization among our proposed EURA and two contrast schemes, and there are five criteria are defined for comparison as Section 4.2.1. Next, our proposed EURA includes three novel mechanisms, and the effect of three mechanisms is analyzed for utilization improvement in Section 4.2.2. Finally, the effect of different traffic packet size is discussed in Section 4.2.3.

\subsubsection{Performance of the proposed EURA scheme}

In Figure 9, Figure 10 and Figure 11, the Max-Rate scheduler might have a better RB utilization due to the concept of maximum value UE's allocation for each RB in a TTI; however, our proposed EURA scheme would have better utilization than the Max-Rate scheduler owing to our proposed EURA scheme that can dynamically use the Eq. (4) for different QCI priority UEs' traffic allocation. The three novel mechanisms can improve the utilization of RB resource allocation. Moreover, our proposed EURA scheme outperforms the EXP/PF scheme owing to the EXP/PF scheme focusing on UEs resource allocation fairness. In Figure 12 and Figure 13, as our proposed EURA scheme has higher utilization, the criteria of delay and total throughput can also have better performance than the other two contrast schemes.

\subsubsection{Effect of three mechanisms}

Based on the Section 4.2.1 simulation results, our proposed EURA scheme outperforms the other two contrasts. We use the gain analysis for the three novel mechanisms (Larger RB capacity first mechanism as Larger $R B$, Lower remaining $R B$ space first mechanism as Lower remaining $R B$, Lower useless $R B$ capacity first mechanism as Lower useless $R B$ in Figure 14 and Figure 15), the gain comparison base is Max-Rate scheduler. To improve the RB utilization allocation, the Lower useless RB capacity first mechanism might be the main influencing factor for our proposed EURA scheme owing to inter-UEs resource allocation. It can also save more RBs' space for the next UE schedule.

\subsubsection{Effect of schedule assignment schemes}

We discuss the impact of changing $\lambda$ and packet size on waste capacity rate, delay, and total throughput when the number of traffic load UEs is the same, i.e., the same $\rho$. We simulated the original packet size as Medium PKT, half of the original packet size as Small PKT, and 1.5 times of the original packet size as Large PKT. In Figure 16 we can find that when packet size value is small, the number of packets in the UE buffer under the same load situation will be high, and the amount of data will be average, i.e., the traffic burstiness is small. Therefore, RB allocation may be required for each TTI. For this reason, as the $\mathrm{RB}$ allocation opportunity increases, more remaining space occurs in each allocation RB. When the packet size is large, it will increase traffic burstiness and at the same time, reduce the need to allocate RB so there is a lower waste capacity rate. However, as the traffic load increases, almost every TTI and RB have a 
chance to allocate UE traffic so the influence of different packet sizes is relatively insignificant.

In Figure 17, we can find that when the packet size value is small, i.e., the traffic burstiness characteristic is small, delay value will also be small; on the contrary, when the packet size value is large, i.e., the traffic burstiness characteristic is larger, and the delay value will also be larger.

In Figure 18, although different packet sizes have a slightly different effect on waste capacity rate and delay, the difference is relatively small so there is no obvious difference in total throughput.

\section{Conclusion}

For 4G/5G mobile wireless network providers, the LTE network has attracted tremendously attention in the world. To increase the efficiency of MAC scheduling in LTE network is still an important and hot issue, and some traditional scheduling schemes have been discussed in some research articles. To improve further mobile network utilization, LTE radio channel capacity is assumed to be dynamic, and eNB can typically select the modulation scheme and code rate depending on a prediction of the DL channel condition, which is based on the UE's CQI report. We propose an EURA scheme with three novel mechanisms, which is considering both UE's CQI states in each RB and overall RBs resource allocation with QoS requirements. The experiment simulation study shows that the RB utilization performance at our proposed scheme is better than the contrasts of EXP/PF and Max-Rate scheduler schemes. In the future, researchers can corporate with other $4 \mathrm{G} / 5 \mathrm{G}$ scheduling schemes to fit mobile users' requirements for different purposes (e.g., fairness, delay guarantee, etc.). These might improve the flexibility of LTE MAC scheduling. Moreover, our proposed three mechanisms can also be applied to other MAC scheduling schemes to improve the utilization of MAC layer resource allocation.

\section{Abbreviations}

LTE: Long Term Evolution; QCI: QoS class indicator; PF: Proportionally Fair; RB: resource block; EURA: Enhanced Utilization Resource Allocation; LTE-A: LTE-Advanced; 3GPP: Third Generation Partnership Project; 5G: fifth-generation; NR: new radio; EPC: Evolved Packet Core; E-UTRAN: Evolved Universal Terrestrial Radio Access Network; MT: maximum throughput; eNB: Evolved Node B; TTI: transmission time interval; RE: resource element; MCS: modulation and coding scheme; QoS: quality of service; UE: mobile user; RBG: RB group.

\section{Declarations}

\section{Availability of data and materials}

Data sharing not applicable to this article as no datasets were generated or analysed during the current study.

\section{Competing interests}

There are no potential competing interests in our paper. And all authors have seen the manuscript and approved to submit to this journal. We confirm that the content of the manuscript has not been published or submitted for publication elsewhere.

\section{Author details}

${ }^{a}$ Department of Sport Management, National Taiwan University of Sport, Taichung City, Taiwan, R.O.C. ${ }^{b}$ Department of Computer Science and Information Engineering, National Chi Nan University, Puli, Nantou County, Taiwan, R.O.C.

\section{Funding}

This work was funded in part by the Ministry of Science and Technology, Taiwan, R.O.C., under grant no. MOST-103-2221-E028-001 and MOST-103-2221-E-164-010. 


\section{Reference}

[1] Sesia, S.; Toufik, I.; Baker, M. P. J. LTE - The UMTS Long Term Evolution: From Theory to Practice, ed.; John Wiley \& Sons Ltd: Chichester, U.K., 2009.

[2] 3GPP, "Radio Access Network; UTRAN Overall Description,". Tech. spec. 3GPP TS 25.401 2011, 10.2.0(2011-06)

[3] 3GPP, "Physical Layer Procedures (Release 10), ". Tech. spec. TS 36.213 V10.0.1 2011.

[4] 3GPP, "Radio Access Network; E-UTRA and E-UTRAN Overall Description,". Tech. spec. 3GPP TS 36.300 2012, 11.1.0(2012-03)

[5] 3GPP, "LTE; Evolved Universal Terrestrial Radio Access (E-UTRA); Physical Layer Procedures,". Tech. Spec. 3GPP TS 36.213 2013, 10.10.0(2013-07)

[6] 3GPP, "Policy and charging control architecture (Release 11),". Tech. spec. TS 23.203 2013, 11.9.0.

[7] 3GPP, "NR; User Equipment (UE) radio transmission and reception; Part 1: Range 1 Standalone,". Tech. Spec. 3GPP TS 38.101-1 2020, 15.11.0(2020-09)

[8] 3GPP, "NR; User Equipment (UE) radio transmission and reception; Part 2: Range 2 Standalone,". Tech. Spec. 3GPP TS 38.101-2 2020, 15.11.0(2020-09)

[9] 3GPP, "NR; User Equipment (UE) radio transmission and reception; Part 1: Range 1 Standalone,". Tech. Spec. 3GPP TS 38.101-1 2020, 16.5 .0 (2020-09)

[10] 3GPP, "NR; User Equipment (UE) radio transmission and reception; Part 2: Range 2 Standalone,". Tech. Spec. 3GPP TS 38.101-2 2020, 16.5 .0 (2020-09)

[11] Puttonen, J.; Kolehmainen, N.; Henttonen, T.; Moisio, M.; Rinne, M.; Lela, P. Dynamic Packet scheduling Performance in UTRAN long term evolution downlink. in Proceeding of IEEE International Symposium on Personal, Indoor and Mobile Radio Communications (PIMRC) 2008, 308-313.

[12] Huang, J.-J.; Lin, W.-K.; Ko, H.-H. A resource allocation algorithm for maximizing packet transmissions in downlink LTE cellular systems. in Proceeding of 2011 IEEE TENCON 2011, 445-449.

[13] Kushner, H. J.; Whiting, P. A. Convergence of proportional-fair sharing algorithms under general conditions. IEEE Transactions on Wireless Communications 2004, 3(4), 1250-1259.

[14] Yang, C.-C.; Chen, J.-Y.; Mai, Y.-T.; Wang, Y.-C. Delay-Sensitive Network Selection and Offloading in LTEA and Wi-Fi Heterogeneous Networks. Journal of Circuits Systems and Computers 2020.

[15] Botella-Mascarell, C.; Perez, J.; Soria, J.; Roger, S. On the Use of Composite Indicators for Mobile Communications Network Management in Smart Sustainable Cities. Applied Sciences 2021, 11(1), 181.

[16] Sun, S.; Yu, Q.; Meng, W.; Li, C. A Configurable Dual-Mode Algorithm on Delay-Aware Low-Computation Scheduling and Resource Allocation in LTE Downlink. in Proceeding of 2012 IEEE Wireless Communications and Networking Conference (WCNC), 2012, 1444-1449.

[17] Chang, I.-C.; Mai, Y.-T. The end-to-end QoS guarantee framework for interworking WiMAX PMP and mesh networks with Internet. Computers and Electrical Engineering 2013, 39, 1905-1934.

[18] Kaleem, Z., Hui, B. \& Chang, K. QoS priority-based dynamic frequency band allocation algorithm for load balancing and interference avoidance in 3GPP LTE HetNet. EURASIP Journal on Wireless Communications and Networking 2014, 185.

[19] Zhou, K., Nikaein, N. Random access with adaptive packet aggregation in LTE/LTE-A. EURASIP Journal on Wireless Communications and Networking 2016, 36.

[20] Lee, Y. L.; Loo, J.; Chuah, T. C.; El-Saleh, A. A. Multi-objective Resource Allocation for LTE/LTE-A 
Femtocel1/HeNB Networks Using Ant Colony Optimization. Wireless Personal Communications 2017, 92, 565-586.

[21] Ahmad, I.; Jang, J.; Chang, K. QoS Priority-Based Mobile Personal Cell Deployment with Load Balancing for Interference Reduction between Users on Coexisting Public Safety and Railway LTE Networks. Electronics 2020, 9, 2136.

[22] Beshley, M.; Kryvinska, N.; Seliuchenko, M.; Beshley, H.; Shakshuki, E.M.; Yasar, A.-U.-H. End-to-End QoS Smart Queue Management Algorithms and Traffic Prioritization Mechanisms for Narrow-Band Internet of Things Services in 4G/5G Networks. Sensors 2020, 20, 2324.

[23] Ismail, S. B.; Ali, D. B. M.; Ya'acob N. Performance Analysis of Uplink Scheduling Algorithms in LTE Networks. Indonesian Journal of Electrical Engineering and Computer Science 2018, 373-379.

[24] Yang, C; He, J. Research on Scheduling Algorithms in Uplink of LTE System. IOP Conference Series: Materials Science and Engineering 2020, 782, 032097.

[25] Ali, S.; Zeeshan, M.; Naveed, A. A Capacity and Minimum Guarantee-based Service Class-Oriented Scheduler for LTE Networks. EURASIP Journal on Wireless Communications and Networking 2013,67.

[26] Jang, H.-C.; Hu, C.-P. Fairness-Based Adaptive QoS Scheduling for LTE. in Proceeding of 2013 International Conference on ICT Convergence (ICTC) 2013, 626-631.

[27] Biernacki, A.; Tutschku, K. Comparative Performance Study of LTE Downlink Schedulers. Wireless Personal Communications 2013, 74(2), 585-599.

[28] Aminu, L. M.; Saidu, I.; Mohammed, A. Downlink Scheduling Algorithms in LTE Networks: A Survey. IOSR Journal of Mobile Computing \& Application 2017, 4(3), 1-12.

[29] Deniz, C.; Uyan, O. G.; Gungor, V. C. On the performance of LTE downlink scheduling algorithms: A case study on edge throughput. Computer Standards \& Interfaces 2018, 59, 96-108.

[30] Nasralla, M. M. A Hybrid Downlink Scheduling Approach for Multi-Traffic Classes in LTE Wireless Systems. IEEE Access 2020, 8, 82173-82186. 


\section{Figure Title and Legend Section}

Figure 1. Diagram of LTE scheduler; Per UE's CQI state for each resource block (RB) should be considered simultaneously in LTE MAC layer resource allocation.

Figure 2. Basic time-frequency resource structure of LTE TTI; An RB is comprised of 84 REs in the case of the normal cyclic prefix length.

Figure 3. Example of traditional resource allocation with QCI priority consideration; Example case of LTE resource allocation in the MT scheduler with the priority queue scheme.

Figure 4. Overview of EURA scheme algorithm; Diagram of our proposed EURA scheme includes these one checking policy and three novel mechanisms.

Figure 5. Example of proposed EURA resource allocation scheme; Example case of our proposed EURA resource allocation scheme.

Figure 6. Notations used in RB capacity estimation; Notations used in the estimation of the all RB capacity for UEs are defined and illustrated.

Figure 7. Simulation architecture; The simulated network environment consists of one LTE-A eNB and 20 UEs.

Figure 8. Hit rate of SchThr larger than BufferUE Data; Estimating the experimental hit rate results for different values.

Figure 9. Simulation result of residual RB's rate; The performance comparison of residual RB's rate for our proposed scheme and the contrasts.

Figure 10. Simulation result of residual capacity; The performance comparison of residual capacity for our proposed scheme and the contrasts.

Figure 11. Simulation result of waste capacity rate; The performance comparison of waste capacity rate for our proposed scheme and the contrasts.

Figure 12. Simulation result of delay; The performance comparison of delay for our proposed scheme and the contrasts.

Figure 13. Simulation result of total throughput; The performance comparison of total throughput for our proposed scheme and the contrasts.

Figure 14. Gain of residual RBs between EURA with three mechanisms and Max Rate; The performance gain of residual RBs between EURA with three mechanisms and Max Rate.

Figure 15. Gain of residual capacity between EURA with three mechanisms and Max Rate; The performance gain of residual capacity between EURA with three mechanisms and Max Rate.

Figure 16. Analysis of waste capacity rate in different packet size situation; The performance analysis of waste capacity rate in different packet size for our proposed EURA scheme.

Figure 17. Analysis of delay in different packet size situation; The performance analysis of delay in different packet size for our proposed EURA scheme.

Figure 18. Analysis of total throughput in different packet size situation; The performance analysis of total throughput in different packet size for our proposed EURA scheme. 
QCl level,

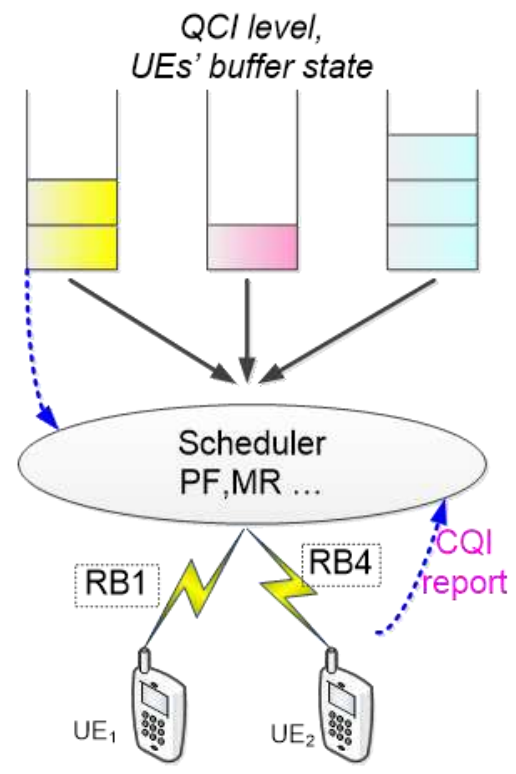

Figure 1. Diagram of LTE scheduler

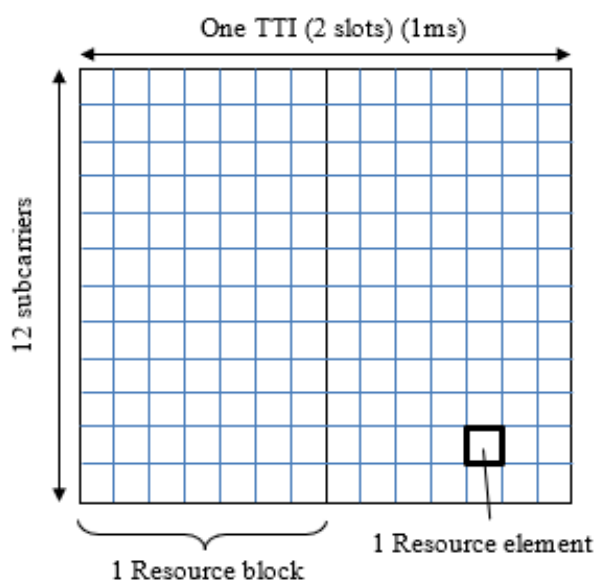

Figure 2. Basic time-frequency resource structure of LTE TTI

Table 1. CQI table by $3 \mathrm{GPP}$

\begin{tabular}{cccc}
\hline CQI index & Modulation & $\begin{array}{c}\text { Approximate } \\
\text { code rate }\end{array}$ & Efficiency (bits/RE) \\
\hline 0 & No Tx & -- & -- \\
1 & QPSK & 0.076 & 0.1523 \\
2 & QPSK & 0.12 & 0.2344 \\
3 & QPSK & 0.19 & 0.3770 \\
4 & QPSK & 0.3 & 0.6016 \\
5 & QPSK & 0.44 & 0.8770 \\
6 & QPSK & 0.59 & 1.1758 \\
7 & 16QAM & 0.37 & 1.4766 \\
8 & 16QAM & 0.48 & 1.9141 \\
9 & 16QAM & 0.6 & 2.4063 \\
10 & 64QAM & 0.45 & 2.7305 \\
11 & 64QAM & 0.55 & 3.3223 \\
12 & 64QAM & 0.65 & 3.9023 \\
13 & 64QAM & 0.75 & 4.5234 \\
14 & 64QAM & 0.85 & 5.1152 \\
15 & 64QAM & 0.93 & 5.5547 \\
\hline
\end{tabular}




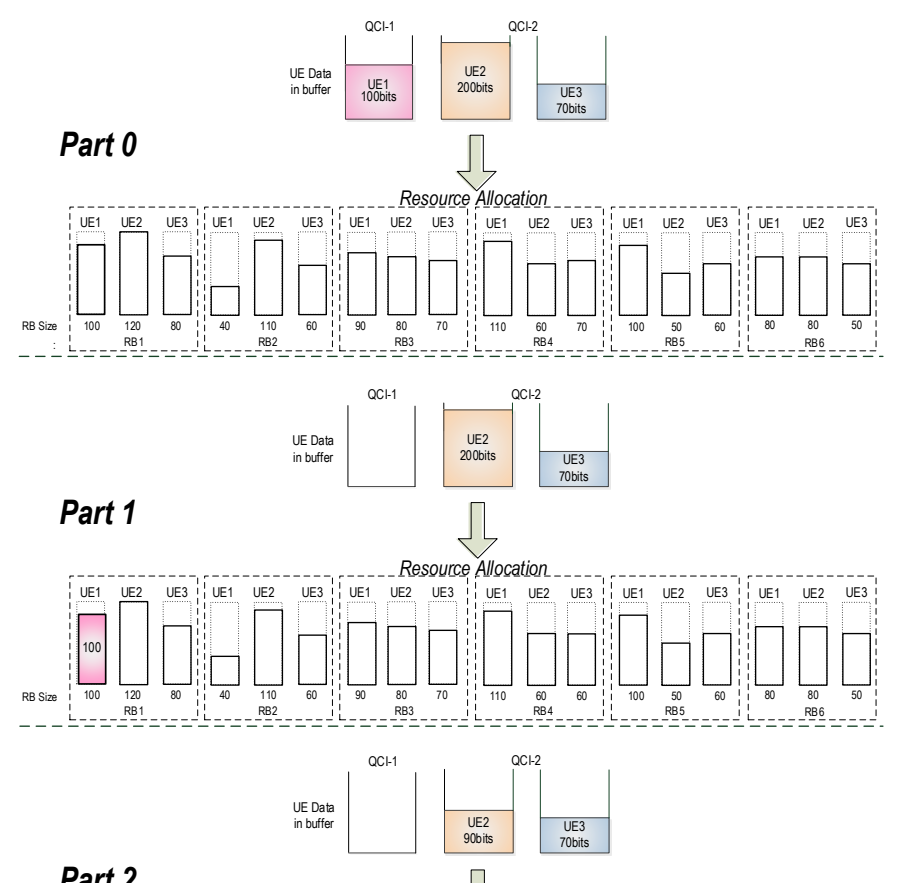

Part 2
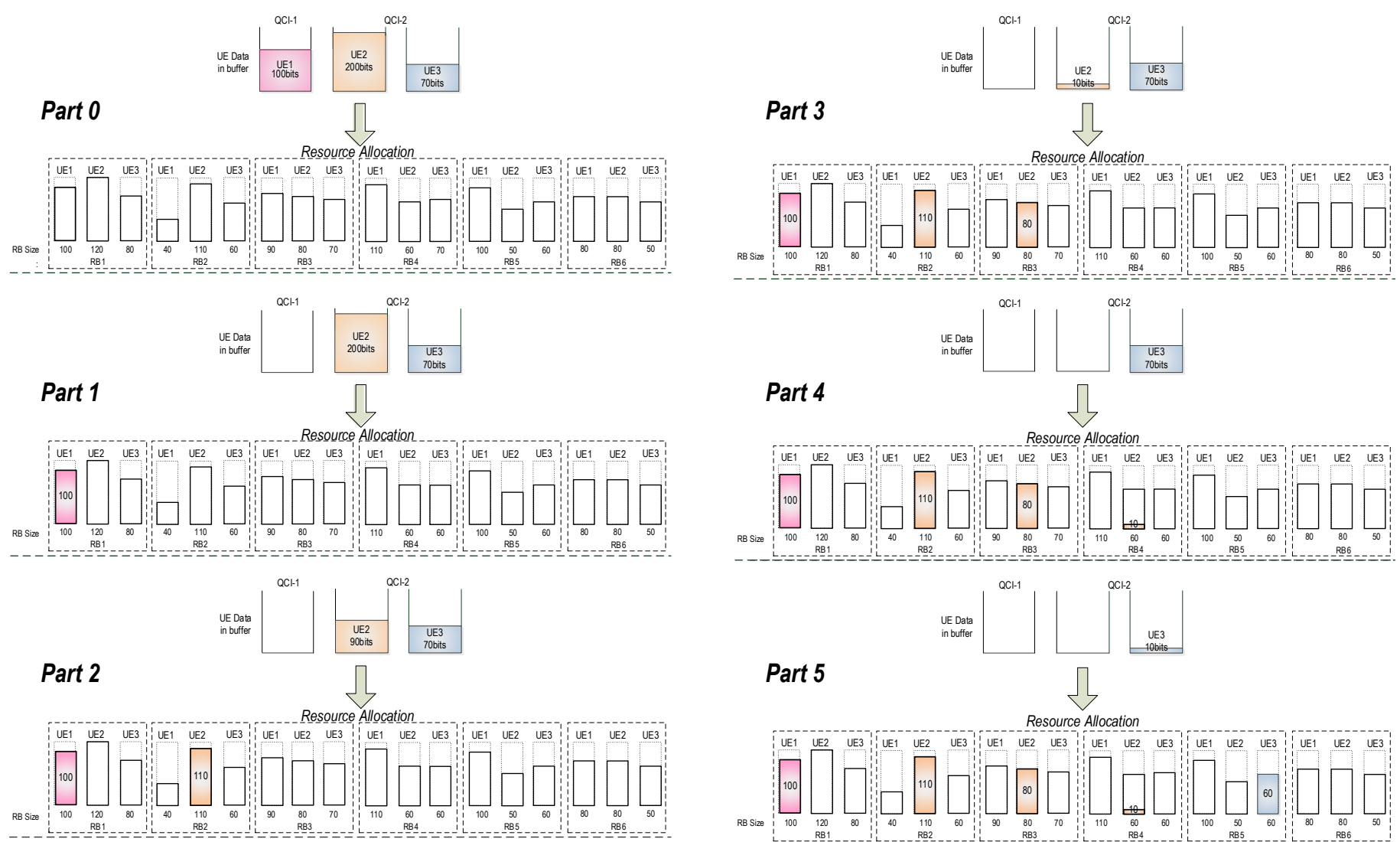

Part 5
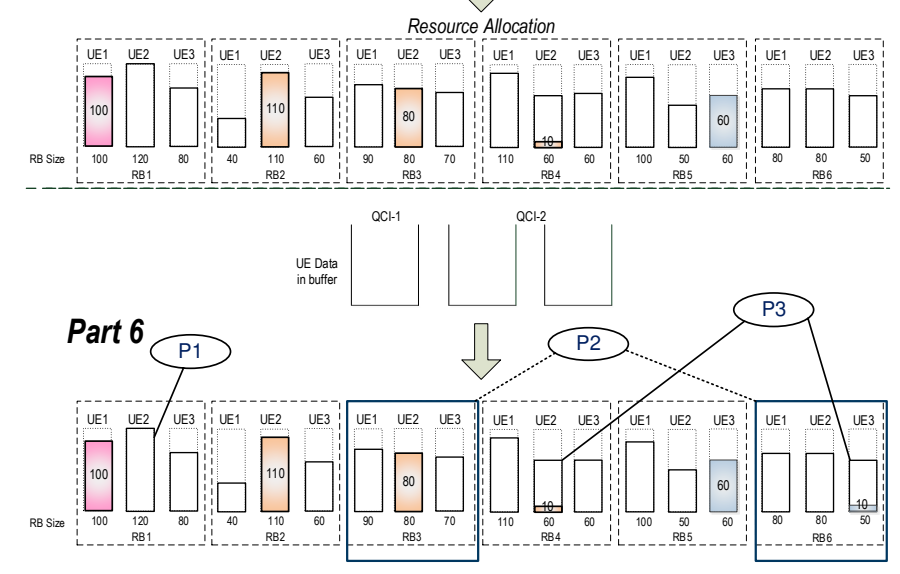

Figure 3. Example of traditional resource allocation with QCI priority consideration 


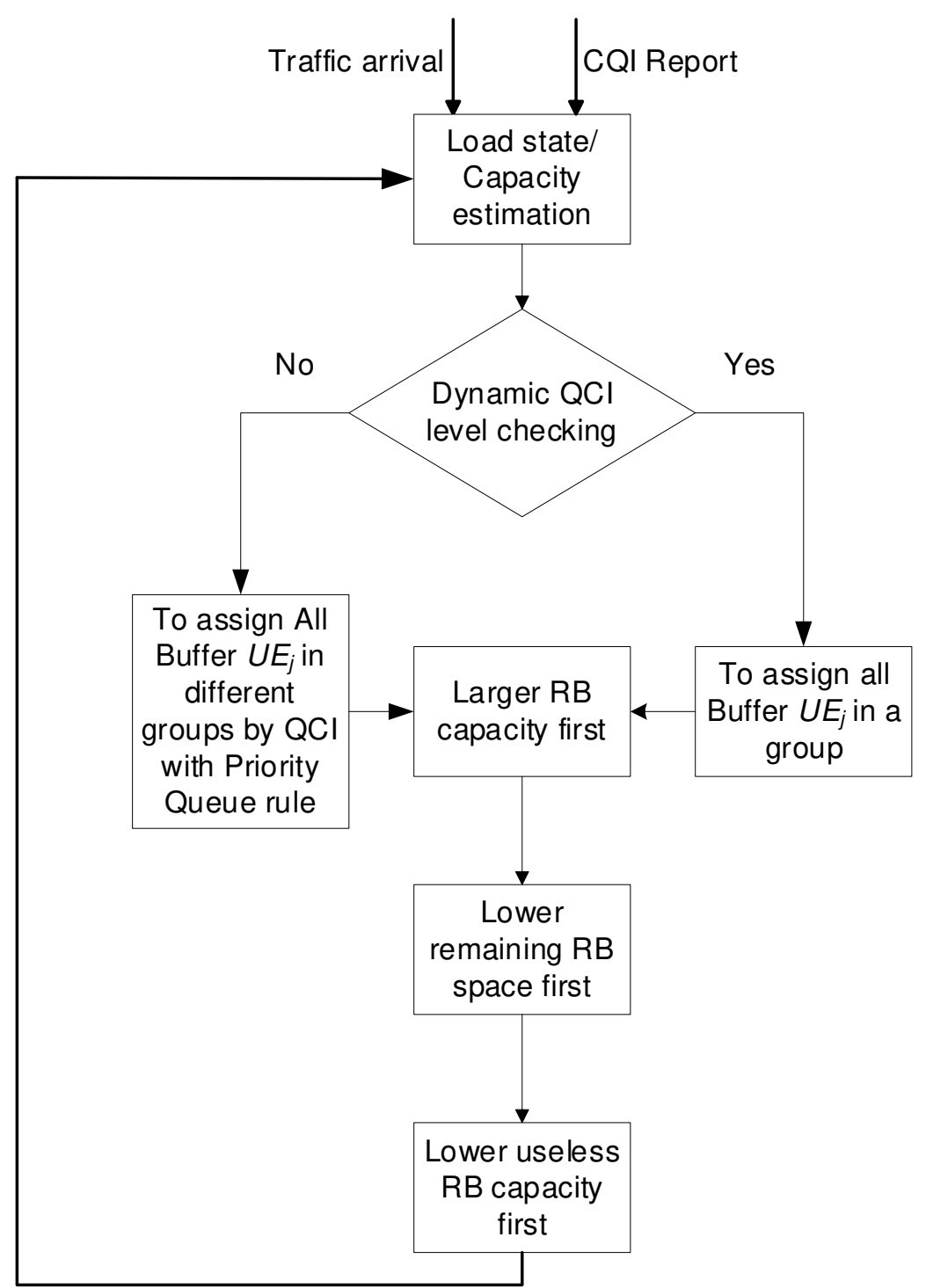

Figure 4. Overview of EURA scheme algorithm 

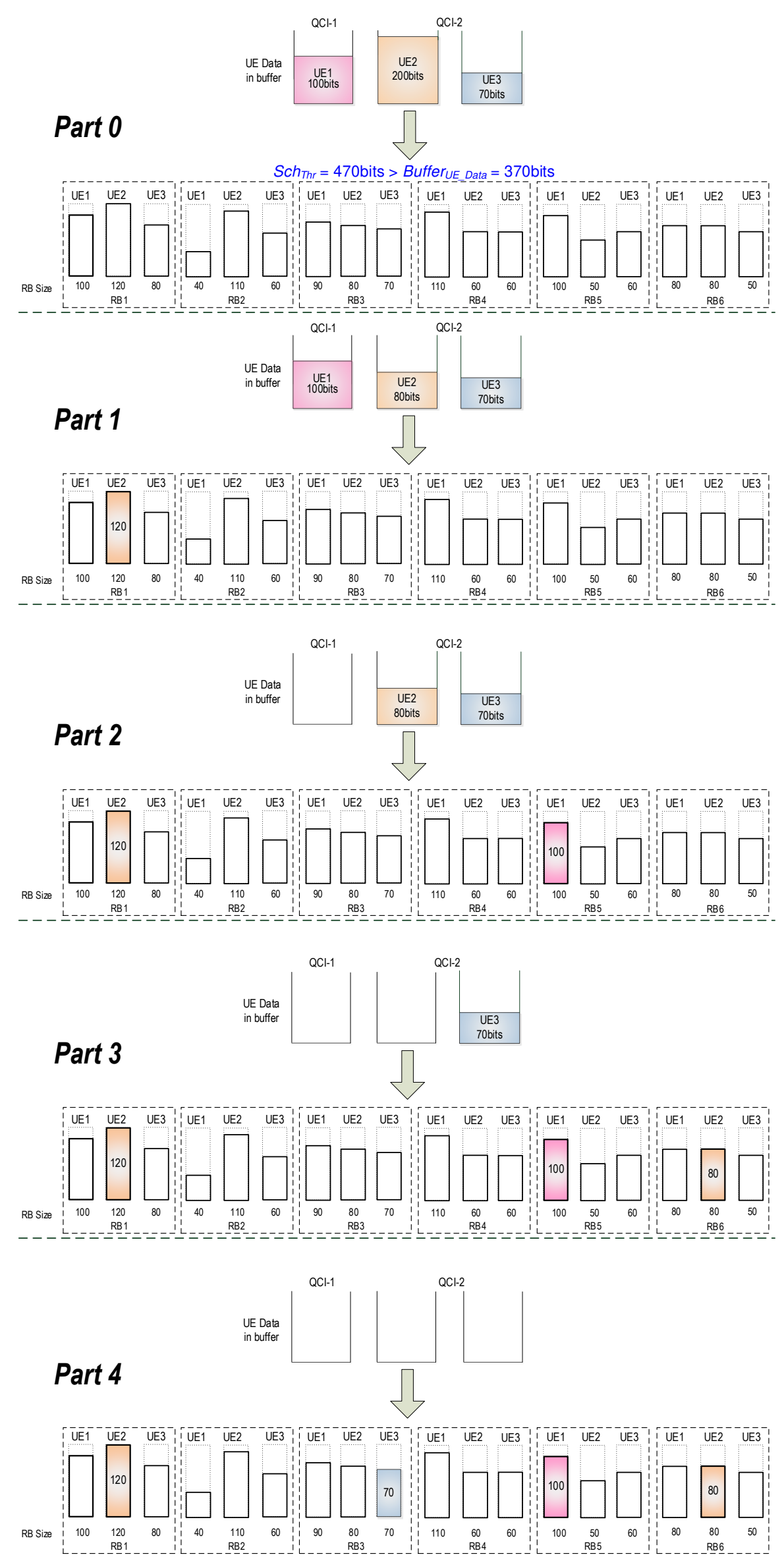

Figure 5. Example of proposed EURA resource allocation scheme 
1 TTI, 14 OFDM Symbols $\left(\mathrm{N}^{\text {TTI }}\right.$ OFDM $\left.=14\right)$

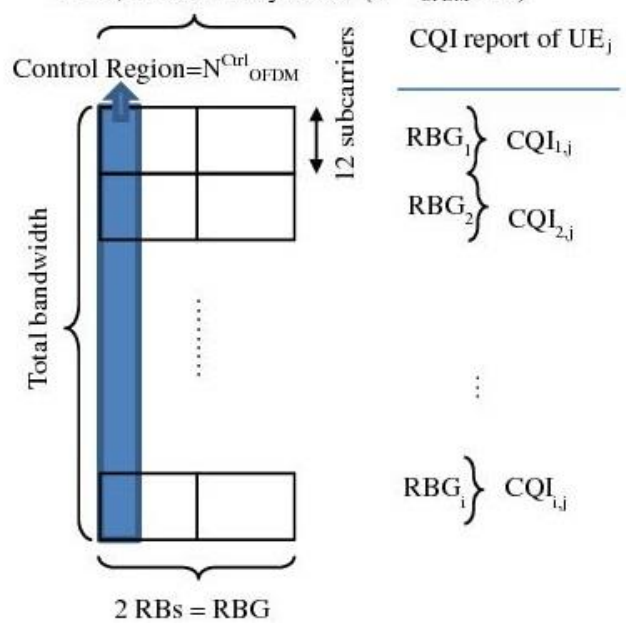

Figure 6. Notations used in RB capacity estimation

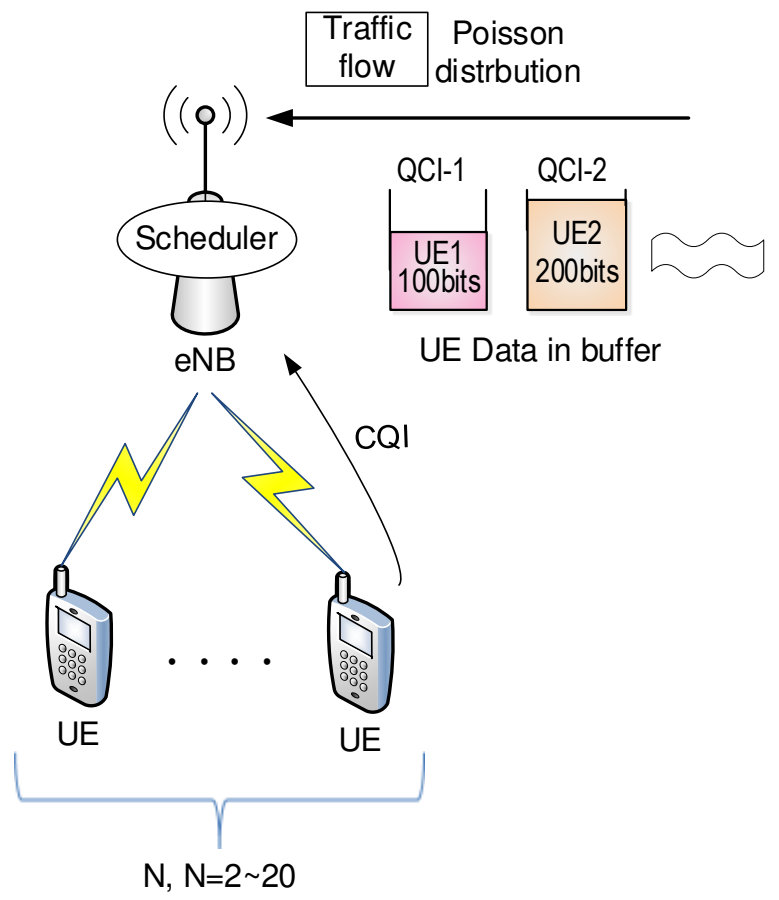

Figure 7. Simulation architecture 


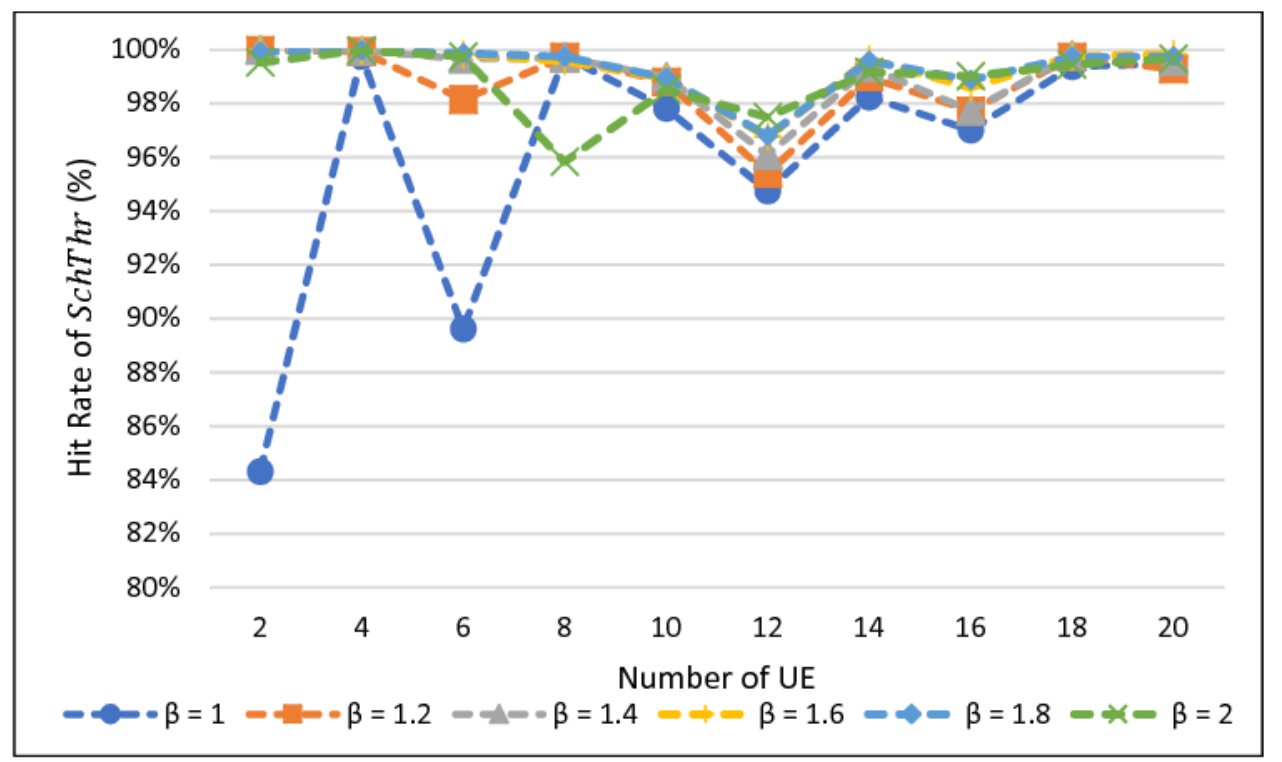

Figure 8. Hit rate of $S c h_{T h r}$ larger than BufferUE_Data

Table 2. Simulation parameters

\begin{tabular}{cc}
\hline Parameter & Value \\
\hline System capacity & $19.3 \mathrm{MHz}$ \\
\# UE & $2 \sim 20$ (equal load) \\
QCI type & QCI-1 QCI-4 \\
\# RB in a TTI & 100 \\
CQI value of RB & Uniform distribution \\
The value of $\beta$ & 1.8 \\
CQI reporting type & All feedback \\
Packet size of audio traffic & 180 bits \\
Packet size of video traffic & 8000 bits \\
\hline
\end{tabular}

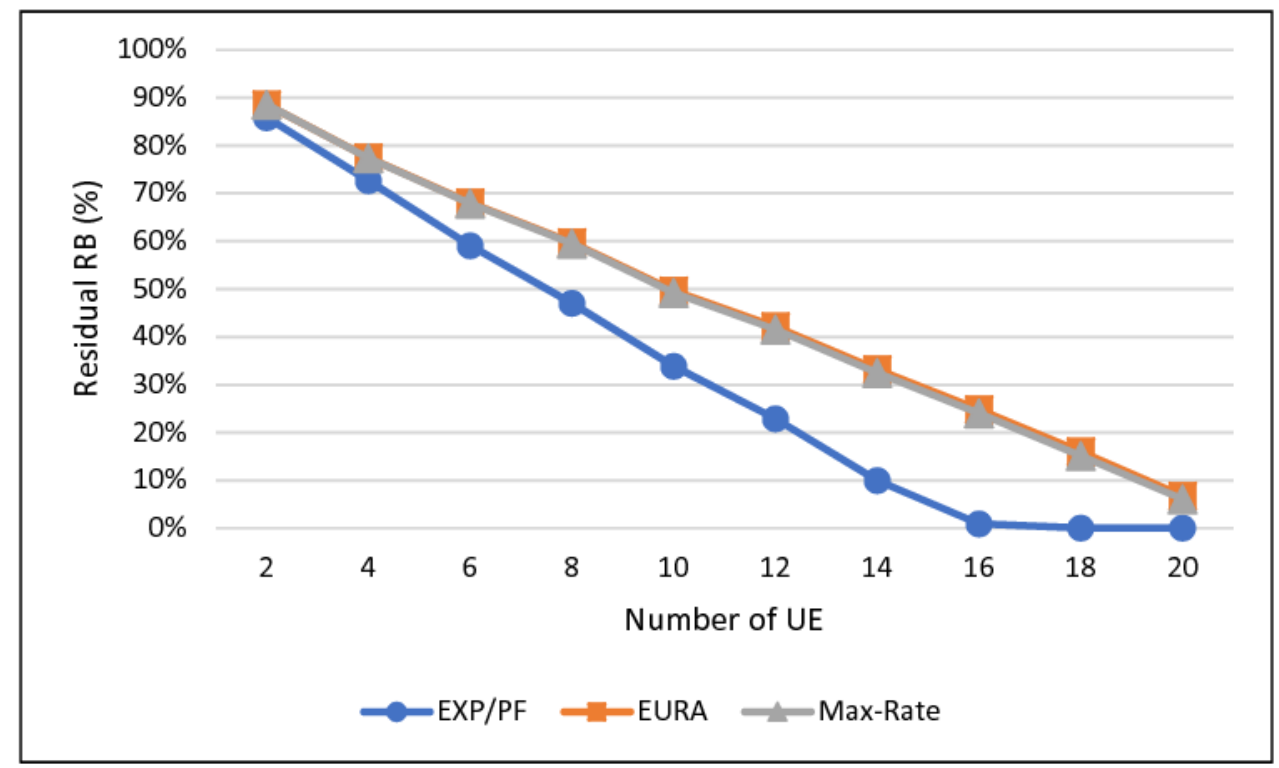

Figure 9. Simulation result of residual RB's rate 


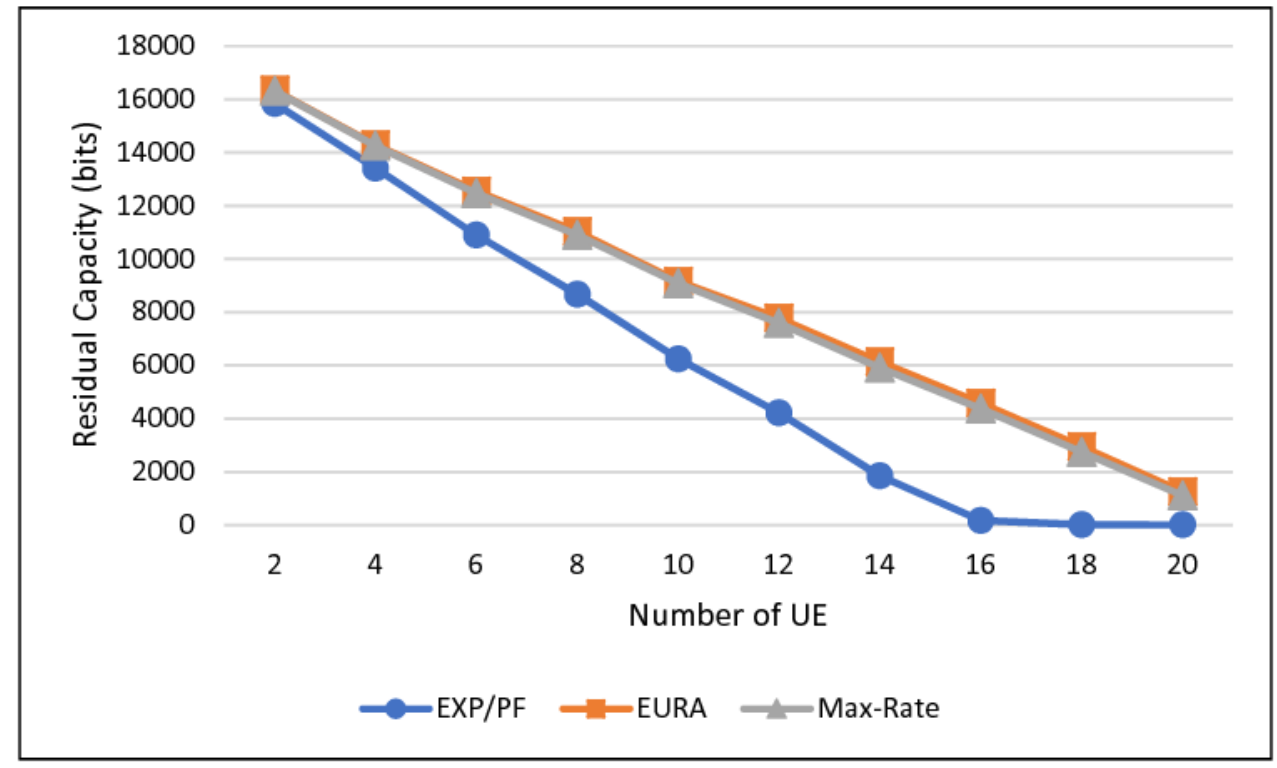

Figure 10. Simulation result of residual capacity

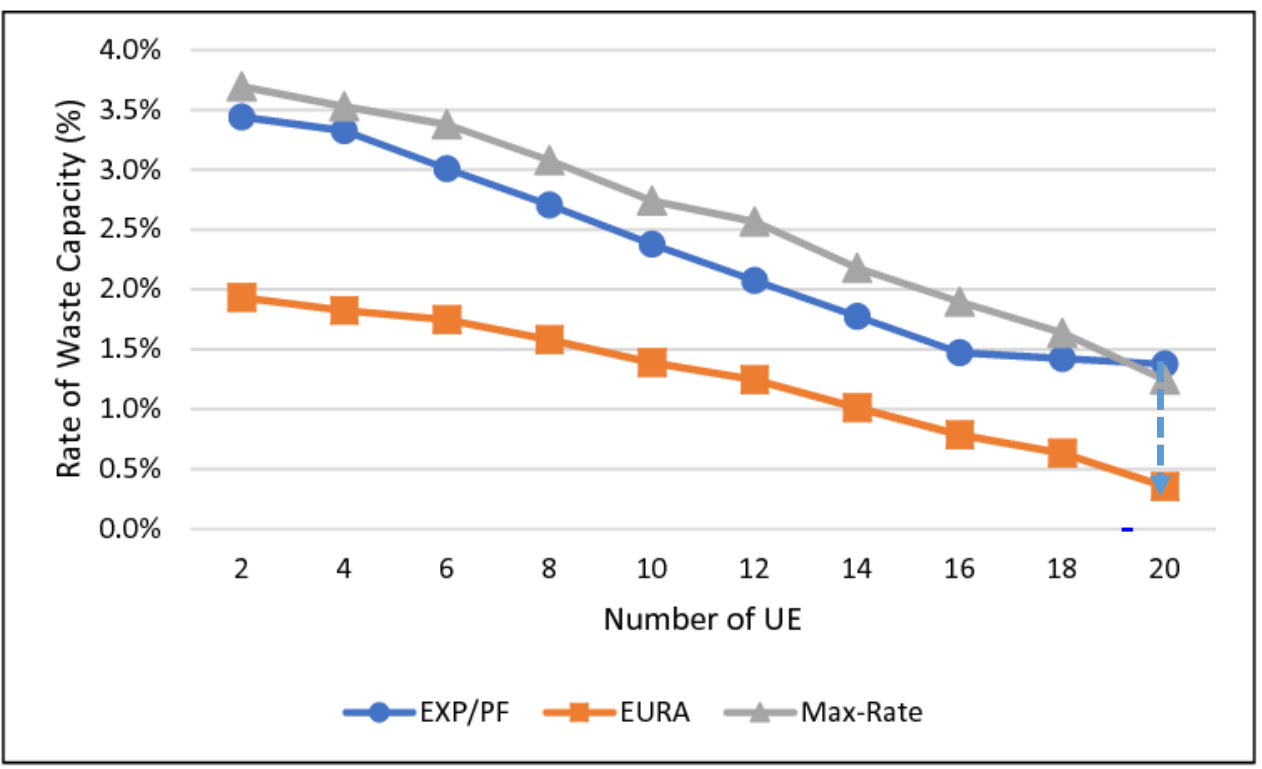

Figure 11. Simulation result of waste capacity rate 


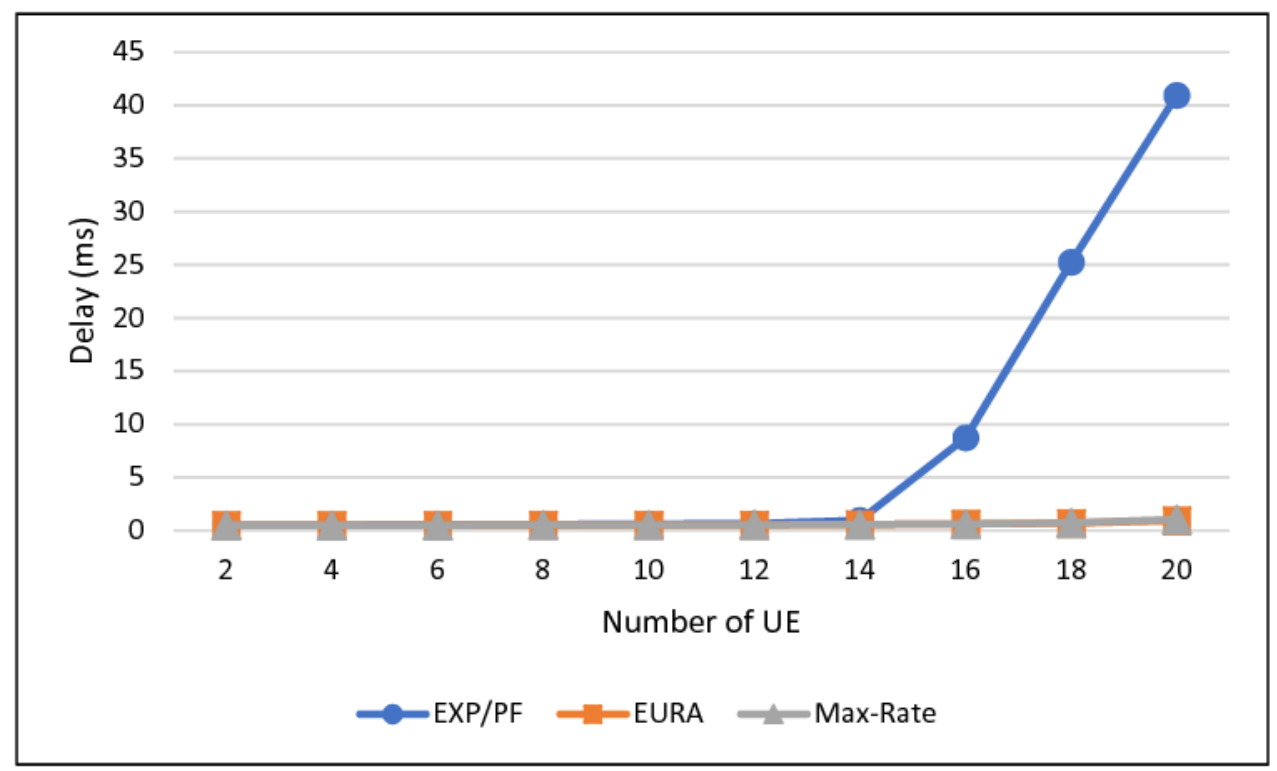

Figure 12. Simulation result of delay

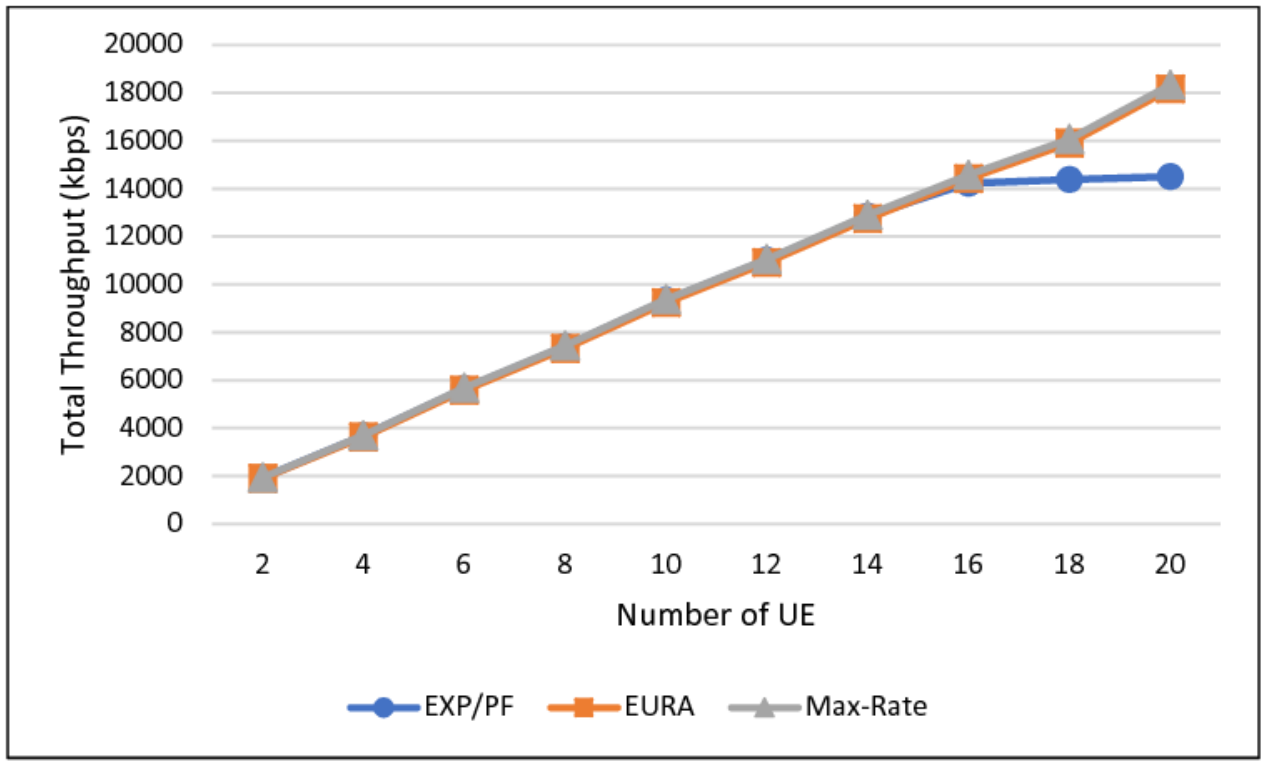

Figure 13. Simulation result of total throughput 


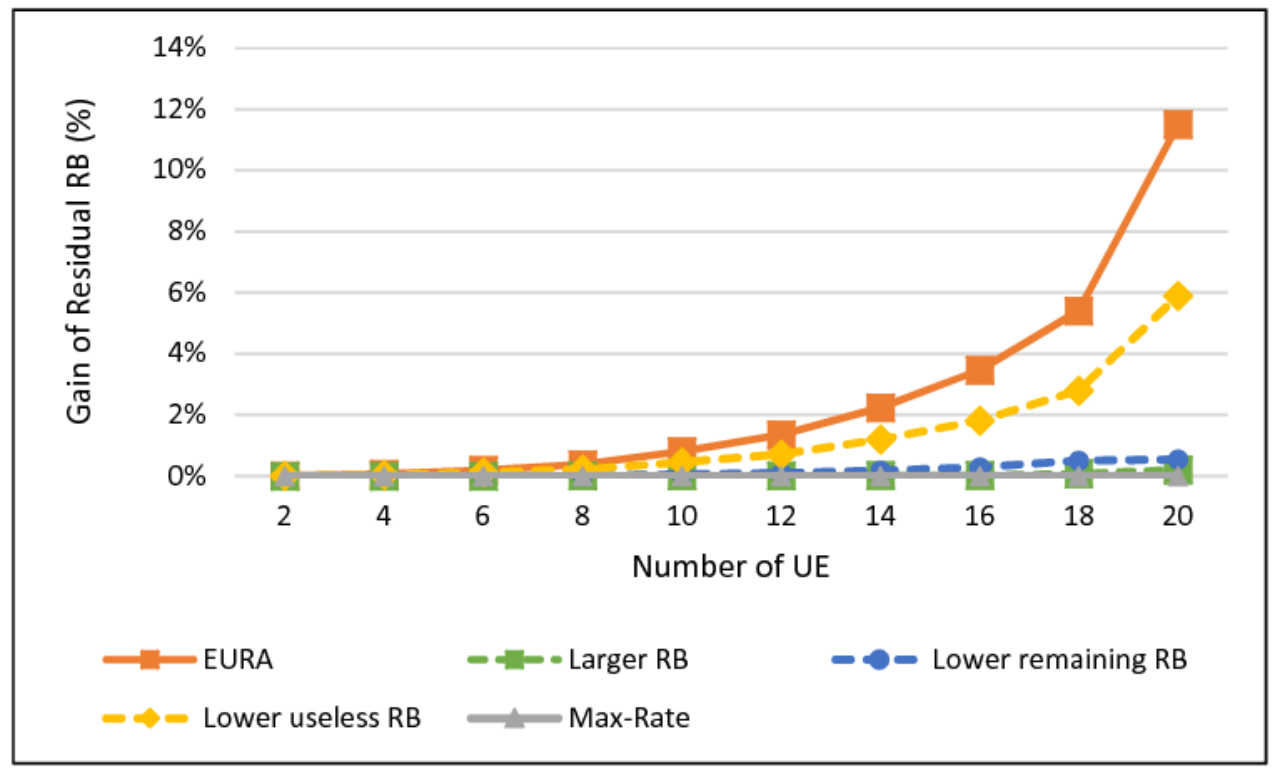

Figure 14. Gain of residual RBs between EURA with three mechanisms and Max Rate

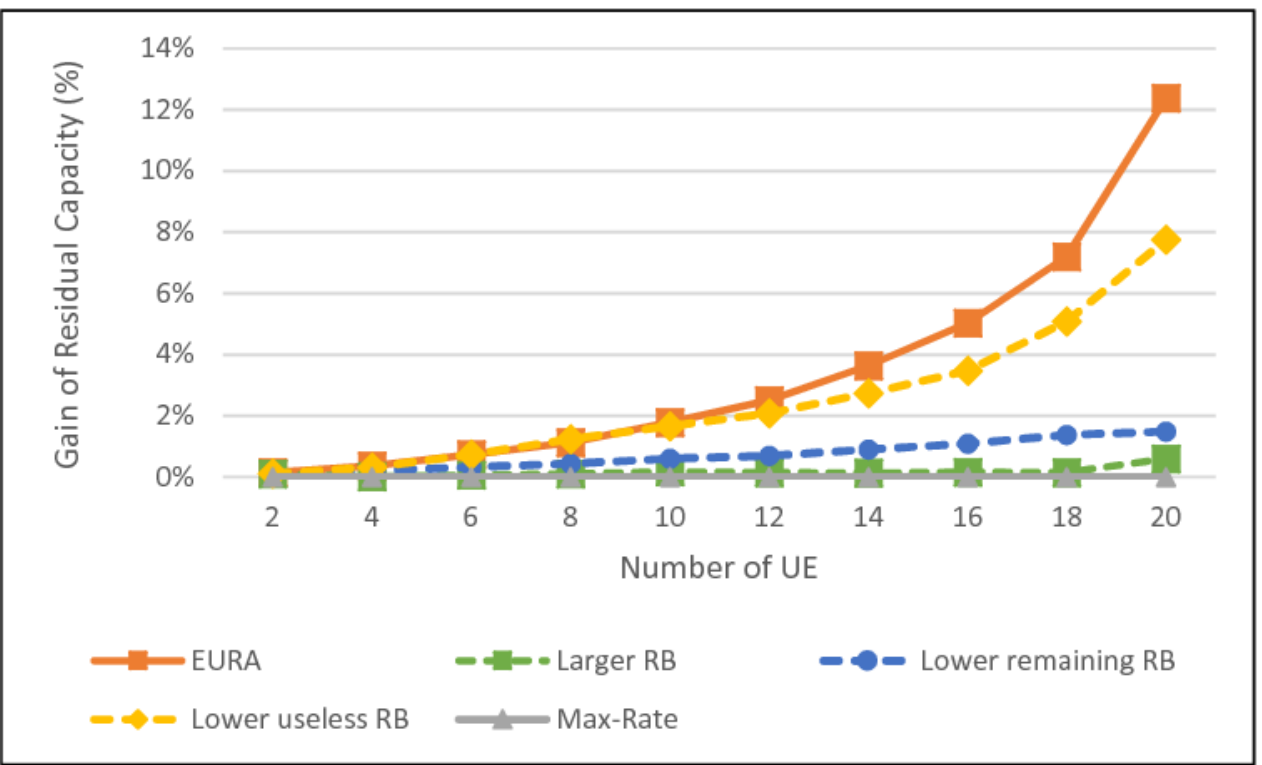

Figure 15. Gain of residual capacity between EURA with three mechanisms and Max Rate 


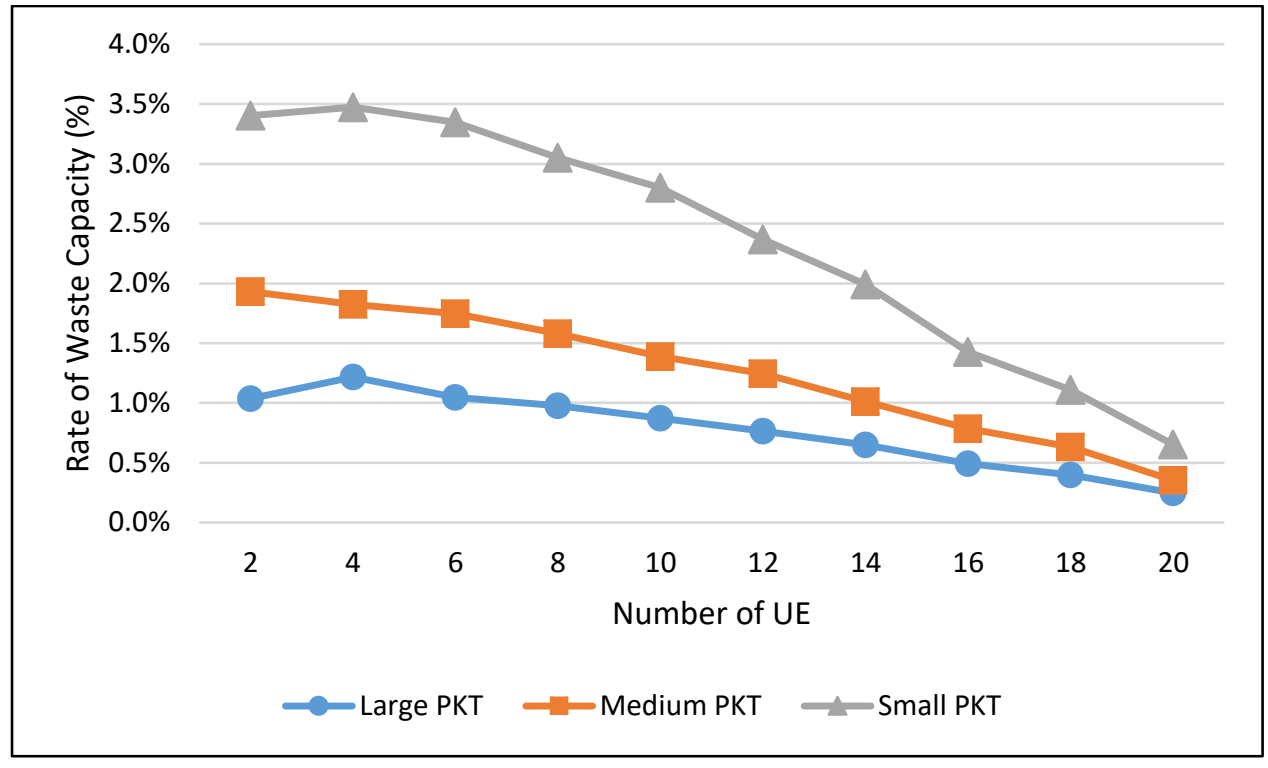

Figure 16. Analysis of waste capacity rate in different packet size situation

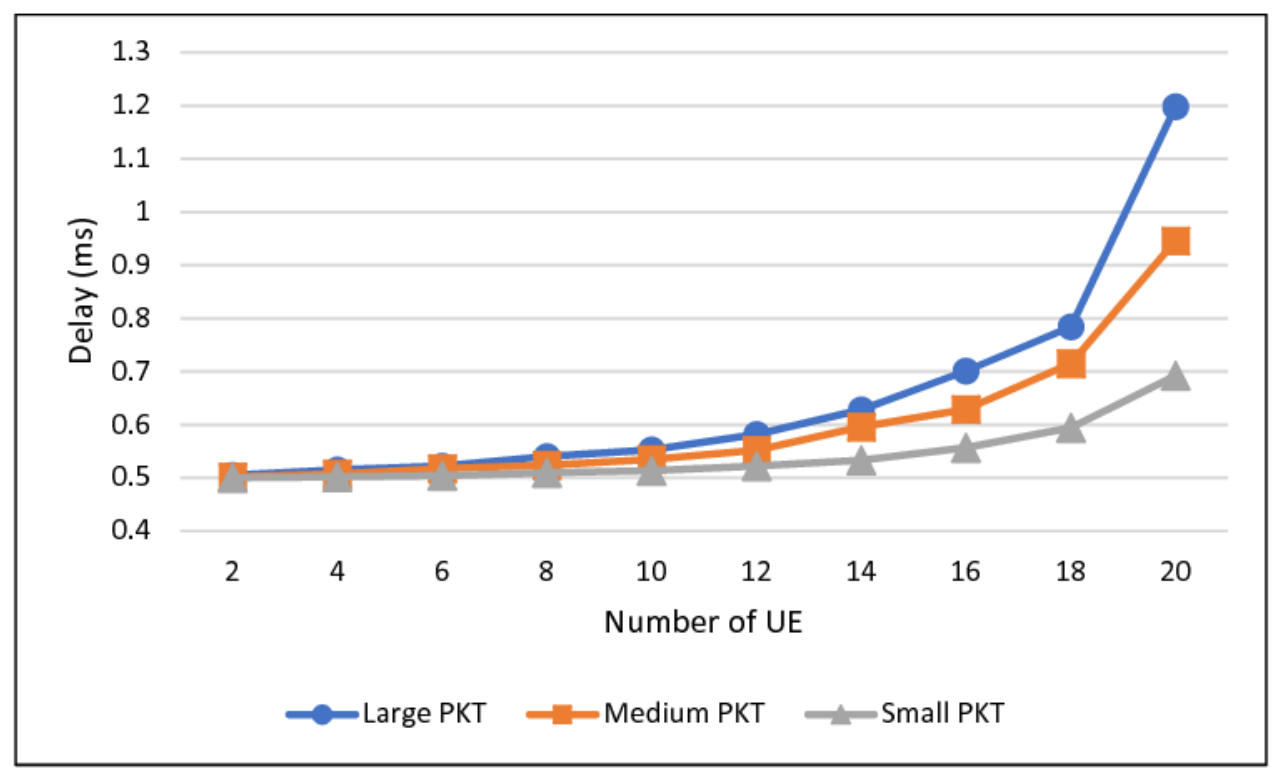

Figure 17. Analysis of delay in different packet size situation 


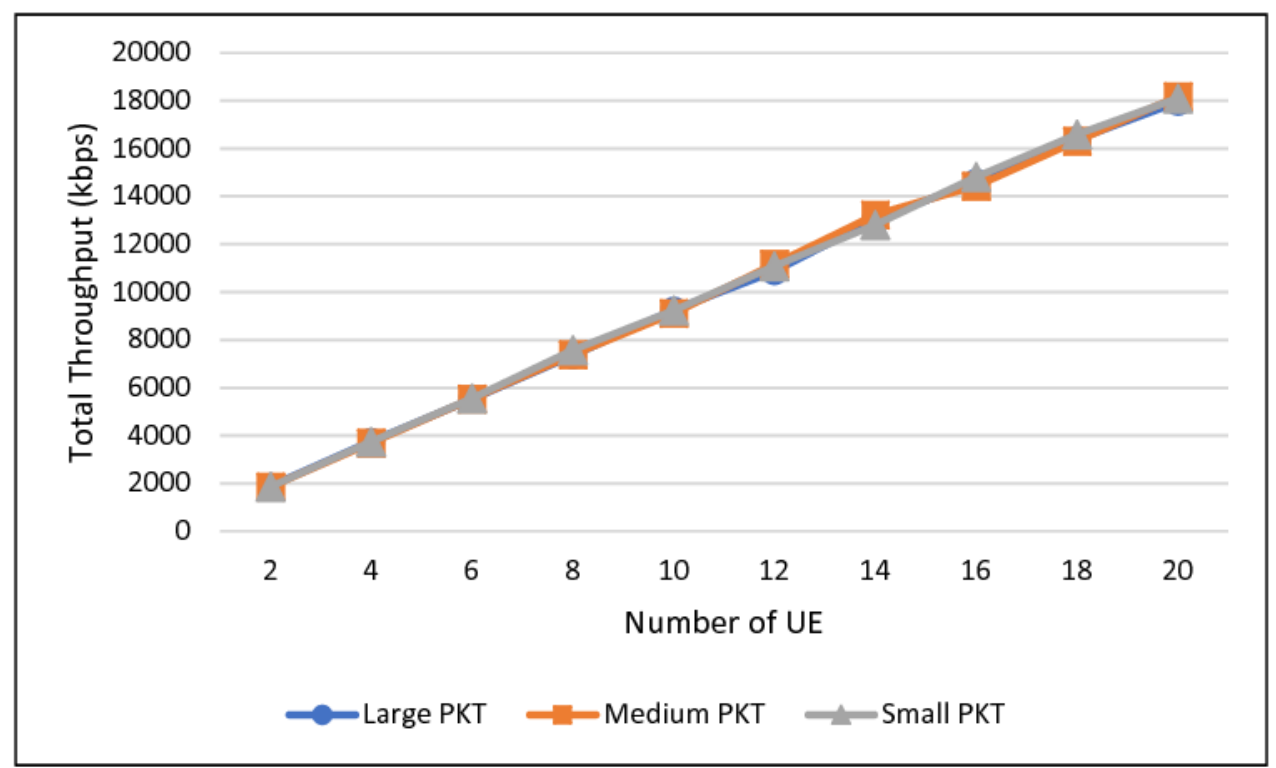

Figure 18. Analysis of total throughput in different packet size situation 